\title{
Didaktischer Einsatz von Online-Fragebögen im GW-Unterricht. Das Beispiel der Wegzugsmotivationen Jugendlicher
}

\author{
* frank.meyer1@tu-dresden.de, Institut für Geographie, Technische Universität Dresden ( $₫$ korresp. Autor) \\ ** robert.vogler@plus.ac.at, Fachbereich Geographie und Geologie \& School of Education, Universität Salzburg
}

eingereicht am: 30.04.2021, akzeptiert am: 28.09.2021

In der humangeographischen Forschung sind Fragebögen ein zentrales Instrument und zunehmend finden gerade OnlineFragebögen besondere Beachtung. Am Beispiel der Beforschung von Wegzugsmotivationen Jugendlicher diskutiert dieser Beitrag im ersten Teil die Arbeitsschritte bei der Erstellung und Auswertung von Online-Fragebögen. Im zweiten Teil wird dies auf die Schulpraxis übertragen, indem einerseits die Potenziale und Andockmöglichkeiten von Online-Fragebögen für den Unterrichtseinsatz skizziert werden und andererseits ein Beispiel einer Unterrichtssequenz vorgestellt wird.

Keywords: Methode, Online-Fragebögen, Wegzugsmotivation, Jugendliche, Unterricht

\section{Educational use of online surveys in the geography classroom. The example of adolescents' migratory considerations}

In human geographic research, questionnaires are a key method, and online questionnaires, in particular, have received increased attention recently. Using the example of researching adolescents' motivation to move away, the first part of this article discusses the working steps to create and evaluate online questionnaires. In the second part, this is transferred to school practice by outlining the potential of online questionnaires for their use in the classroom. Then, a learning environment supported by an online survey is presented, which makes the topic of potential migration motivation of young people interactively addressable.

Keywords: method, online surveys, migration motivation, adolescents, classroom

\section{Wegzugsmotivationen Jugendlicher in ländlichen Regionen beforschen: Welche Methoden stehen uns zur Verfügung?}

Die Frage, welche Gründe aus der Sicht von Jugendlichen nach Ihrem Schulabschluss für das Verlassen von oder Bleiben in ihrem Heimatort sprechen, bzw. was letztlich zu Wegzügen führt, ist in der deutsch- und englischsprachigen geographischen Mobilitätsforschung Gegenstand wiederholter empirischer Erhebungen. Während im Allgemeinen karriere- und ausbildungsbezogene Gründe häufig als Triebfedern regionaler Abwanderungsprozesse bei Jugendlichen thematisiert werden (bspw. Hansen \& Aner 2017), wurden innerhalb der letzten 10 Jahre vermehrt auch subjektive Rationalisierungsprozesse für Migrationserwägungen in den Fokus genommen. Diese Ansätze zentrieren eher den Entscheidungsfindungsprozess, sowie nicht- materielle, emotionale und psychologische Aspekte (bspw. Collins et al. 2014; Carling \& Collins 2018). Infolge dieser Forschungsbeiträge wurde offenbart, wie stark Gehen und Bleiben auch diskursiv aufgeladen sind und häufig mit Aspekten von Selbstverwirklichung bzw. „Zurückbleiben“ verwoben werden (bspw. Meyer et al. 2016; Wiest 2016). Darüber hinaus spielen unvollständige und selektive Informationen über regionale Möglichkeiten oder die tatsächlichen Bedingungen des Zielortes, sowie die biographischen Mobilitätserfahrungen der eigenen Eltern eine große Rolle in der Ausprägung der Migrationserwägungen Jugendlicher (bspw. Leibert 2016). Ein negatives Binnenimage der Heimatregion bei deren Einwohner*innen und ggf. prekäre sozioökonomische Bedingungen in der Heimatregion verstärken hierbei die Wahrscheinlichkeit bei Jugendlichen, wegziehen zu wollen (bspw. Meyer 2017). 
Möchte man dieses Thema empirisch beforschen, so kommen unterschiedliche Ansätze infrage und beeinflussen hinsichtlich ihrer Wahl und individuellen Durchführung maßgeblich den potentiellen Erkenntnisgewinn (vgl. Meyer et al. 2019): Im Rahmen von Interviews sind Einzelgespräche mit Jugendlichen über ihre Pläne möglich. Jedoch hängt es von der Vertrauensbeziehung zwischen interviewender und interviewter Person ab, inwieweit hier ein Zugriff auf sehr persönliche und emotionale Themen möglich ist. Im Rahmen von Gruppendiskussionen ist eine breitere empirische Basis gegeben und durch den Diskussionscharakter ist potentiell der Zugriff auf regionale Diskursmuster möglich, die letztlich zur Herausbildung von regionalen Abwanderungskulturen führen (vgl. Meyer \& Leibert 2021). Beide Methoden könnten innerhalb von Mixed-Methods-Forschungsdesigns (vgl. Callegaro et al. 2015: $37 \mathrm{ff}$.) auch als explorative und/ oder hypothesengenerierende Schritte mit geringen Stichproben dienen, um nachfolgend auf deren Basis standardisierte Befragungen mittels Fragebögen (vgl. Baur \& Blasius 2014: 600ff.) entwickeln zu können. Diese eignen sich insbesondere dann, wenn eine etablierte Wissensbasis zu generellen Gründen und Funktionsweisen von Migrationserwägungen gegeben ist (bspw. durch vorhandene Forschungsliteratur), und nun eine breitere Stichprobe, eine spezifische Subgruppe, eine spezifische Untervariable oder ein anderer Kontext (bspw. eine andere Region) daraufhin befragt werden soll. Gleichermaßen sind auf der Basis von Ergebnissen standardisierter Befragungen vertiefende qualitative Erhebungen möglich. Bei der Wahl einer Erhebung mittels Fragebogen stellt sich zudem die Frage, ob dieser online oder in Papierform ausgegeben wird, was jeweils bestimmte Vor- und Nachteile hat (siehe Kap. 2).

Basierend auf dem Beispiel der „Wegzugsmotivationen Jugendlicher" ist das Ziel dieses Artikels, die Erhebungsmethode des Fragebogens in seiner Konzeption initiativ zu beschreiben, dabei die Potentiale von Online-Fragebögen zu diskutieren und anschließend danach zu fragen, wie diese Methode im Unterricht fachdidaktisch gewinnbringend adaptiert werden kann. Im Folgenden wollen wir zunächst klären, welche Arbeits- und Reflexionsschritte generell im Prozess der Fragebogenerstellung praktisch zu gehen sind und eine mögliche Anwendung im Rahmen des Schulunterrichts insbesondere für jene Altersgruppen konzeptualisieren, für die die Frage von Wegzug oder Verbleib in ihrer Heimat ein relevantes Thema ist: die Sekundarstufe 2. Dabei wird aufgrund der recht einfachen Operationalisierung und Echtzeitverfügbarkeit erster Ergebnisse (siehe Kap. 3) die Verwendung eines Online-Fragebogens angedacht.

\section{2 (Online-)Fragebögen als humangeogra- phische Forschungsmethode: Arbeits- und Reflexionsschritte}

Während die Coronapandemie ab 2020 die Wahl empirischer Erhebungsmethoden häufig - in Richtung digitaler Erhebungen - vereinfacht, bringt die zunehmende Verlagerung von Erhebungen in den digitalen Raum (vgl. Welker \& Wünsch 2010 für eine Systematisierung; Taddicken 2013 für einen Überblick) jedoch nicht nur Vorteile, sondern auch Nachteile mit sich (vgl. Wagner \& Hering 2014: 662f.; Callegaro et al. 2015: $18 \mathrm{ff}$.): Kontaktversuche zur Teilnahme gehen häufig in einer Vielzahl von Anfragen unter und neben einer Überforschtheit von bestimmten Regionen oder Bevölkerungsgruppen (vgl. Wagner \& Hering 2014: 662) kann sich auch durchaus eine Gleichgültigkeit bei möglichen Teilnehmenden einstellen. Eine persönliche Durchführung bringt hier die Möglichkeit, potentielle Teilnehmende stärker zur Teilnahme oder zum Durchhalten bei der Fragebogenbearbeitung motivieren zu können, während ein Online-Fragebogen zweifellos arbeitsökonomisch geringere Ressourcen benötigt - im Bereich Verbreitung, hinsichtlich der maximal möglichen Anzahl Teilnehmender oder bezüglich flankierender Kosten für Druck und Versand. Gleichzeitig sind jedoch neue Fragen zu klären bspw. hinsichtlich der Verbreitung des Zugangs zur Online-Erhebung, sowie hinsichtlich der Datenspeicherung, -weiterverarbeitung und -archivierung. Ein bewusstes Abwägen dieser Fragen muss jedoch nicht unbedingt eine Hürde sein, sondern kann vielmehr Schüler*innen bei einer aktiven Adaption im Unterricht genau für diese Aspekte sensibilisieren. Da für Online-Fragebögen aktuell eine Vielzahl von webbasierten Werkzeugen zur Verfügung stehen ${ }^{1}$, bieten sich diese Methoden insbesondere im didaktischen Bereich zur Nutzung an (siehe Kap. 3).

Grundlegende Gütekriterien und damit grundlegende Ansprüche an standardisierte Befragungen (vgl. Reinecke 2014: 612f.; Kühne 2013: $34 \mathrm{ff}$.; Moosbrugger \& Kelava 2012: 8 ff.) gelten unabhängig von der Wahl digitaler Mittel und umfassen insbesondere Objektivität als Unabhängigkeit der Ergebnisse von Interviewern, Reliabilität als Reproduzierbarkeit der Ergebnisse und Validität als Genauigkeit der Messung. Nach der Wahl des Forschungsgegenstands (bspw. Wegzugsmotivationen Jugendlicher) und der Methodenentscheidung erfolgen i.d.R. nachfolgend

\footnotetext{
bspw. LimeSurvey, SurveyMonkey, Google Formulare oder Microsoft Forms im Education Bundle von Microsoft; eine Systematik findet sich bei Callegaro et al. (2015: 215ff.) Es sei jedoch darauf verwiesen, dass diese teilweise mit unterschiedlichen datenschutzbezogenen Standards operieren, die es im Einzelfall zu betrachten gilt.
} 
spezifische Arbeitsschritte der Fragebogenerstellung, die wir im Folgenden skizzieren möchten²:

\section{Schritt 1: Konkretisierung der Frage und der Rahmenbedingungen}

Am Anfang der inhaltlichen Fragebogenkonzeption steht die Formulierung einer grundlegenden Fragestellung, anhand welcher - in deskriptiver Perspektive (Steiner \& Benesch 2018: 42) - bezüglich eines spezifischen sozialen Phänomens Erklärungen gesucht werden, bzw. nach dem explikativen Ansatz (ebd.) Hypothesen formuliert und mittels der erhobenen Daten überprüft werden sollen (Kühne 2013: 24). Dieser Schritt grenzt die Ausrichtung und Anzahl aller möglichen Aspekte des Themas und der daraus ableitbaren Fragebogenelemente (sog. items) ein.

Neben der inhaltlichen Ausgestaltung ist zudem eine Abschätzung hinsichtlich zeitlicher und finanzieller Rahmenbedingungen für die Fragebogenerstellung und -durchführung notwendig. Die Erarbeitung eines groben Zeitplanes stellt dabei einen häufig übersehenen und trotzdem essentiellen Arbeitsschritt dar. Dabei sind Fragen zu beantworten, wie z. B.:

- In welchem Zeitraum sollen Erstellung, Teilnehmer*innen-Rekrutierung, Durchführung und Auswertung erfolgen?

- Welche Ressourcen stehen zur Verfügung (a) bezüglich der Verbreitung des Aufrufs zur Teilnahme, (b) bezüglich der Überführung der Antworten in Auswertungsprogramme, und (c) hinsichtlich der Nutzung fortgeschrittener Kommunikationstechnologien (bspw. von Smartphones; Callegaro et al. 2015: $192 \mathrm{ff}$.)?

- In welchem Ausmaß sind Tests des Fragebogens möglich?

- Welche Zielgruppe soll befragt werden und was sind geeignete Rekrutierungsstrategien, -mittel und -orte?

- Welche Stichprobengröße wird angestrebt und welche Zusammensetzung soll diese haben? (vgl. Wagner \& Hering 2014: 664f.; Callegaro et al. 2015: 40 ff.; als grundlegende Einführung hierzu Jandura \& Leidecker 2012: 61 ff.)

\section{Schritt 2: Konzeption eines Fragebogenentwurfs}

Bei jeder Art von Fragebogen muss bestmöglich sichergestellt werden, dass - wenn keine interviewende Person präsent ist - die Beantwortung trotzdem sorg-

\footnotetext{
Hierbei ist zu beachten, dass alle diese Schritte sowohl für Online- als auch klassische Fragebögen gelten und an den jeweils relevanten Stellen auf spezifische Umstände für Online-Fragebögen hingewiesen wird.
}

fältig erfolgt. Zusätzlich zum eigentlichen Fragenteil sind deswegen ergänzende Informationen notwendig (siehe unten). Während ein Titelblatt (vgl. Porst 2014: 33 ff.) und ein einheitliches Design eher ästhetische und motivierende Vorteile haben und mehr Übersicht und Vertrauen seitens der Antwortenden hervorrufen (Hollenberg 2016: 8), ist ein einleitender Informationsteil von größerer Bedeutung: Hier sind die Hintergründe der Befragung, deren Zielstellung, sowie rechtlich besonders bedeutsame Anonymisierungs- und Datenschutzinformationen zu geben (vgl. auch RatSWD 2017). Auch ist es notwendig, dass die Antwortenden Informationen erhalten, wie der Fragebogen inhaltlich aufgebaut ist, ggf. welche Fragearten vorkommen, und - falls notwendig - konkrete Anweisungen und Hilfestellungen zum Ausfüllen bekommen (vgl. Porst 2014: $47 \mathrm{ff}$.). Weiterhin ist es (gerade bei Online-Fragebögen) wichtig, darüber aufzuklären, was nach dem Abschluss des Fragebogens geschieht (bspw. eine Information, wann die Daten erfolgreich übermittelt wurden und der Browser geschlossen werden kann) bzw. an wen sich Antwortende vor, während oder nach der Durchführung der Befragung für Hilfestellung oder Feedback wenden können.

Der eigentliche Hauptteil des Fragebogens besteht aus den inhaltlichen Fragen, die sich aus der Forschungsfrage und dem gewählten Forschungsdesign ergeben und welche im Rahmen einer Operationalisierung, d.h. dem Akt des Messbar-Machens, systematisch hergeleitet werden (ausführlich hierzu Steiner $\&$ Benesch 2018: 50 ff.), und folgt dann u.a. folgenden Grundprinzipien (vgl. auch Porst 2014: 137 ff.):

- Verständlichkeit: Ziel aller Informationen und Fragen ist immer, dass Antwortende die Intention und Zielstellung sprachlich und inhaltlich verstehen. Dies bedeutet teils, dass auf lange Fragen möglichst verzichtet werden sollte.

- Flow: Antwortende sollten anfangs einige - gern allgemein themenbezogene - Eisbrecherfragen beantworten, bevor schwierige oder sensible Fragestellungen fokussiert werden, die potentiell Nicht-Antworten provozieren würden (vgl. Hollenberg 2016: 21 f.).

- Filtern: Nicht alle Fragen müssen von allen Personen beantwortet werden; manchmal sind Antworten von Untergruppen (bspw. nach Schulart, Alter, Geschlecht, Bildungsgrad der Eltern, Einkommen) interessant, deren Relevanz aus dem Forschungsstand hergeleitet werden kann. In solchen Fällen können Filterfragen zum Einsatz kommen (vgl. auch Porst 2014: 155 ff.).

- Datensparsamkeit: Personenbezogene Daten sollten nur in dem Maße erhoben werden, zu dem sie zwingend für die Erhebung notwendig sind. In solchen Fällen sollten sie aus befragungstakti- 
schen Gründen am Ende abgefragt werden, wenn eine Antwortverweigerung unwahrscheinlich ist bzw. im schlimmsten Fall keinen Befragungsabbruch hervorrufen würde.

\section{Schritt 3: Konkrete Fragen konzipieren}

In der Folge unterscheiden Jonkisz et al. (2012) bei der Fragebogen- und Itemkonstruktion zwischen intuitiver Konstruktionsstrategie, wenn noch wenig theoretisches Wissen zum Gegenstand der Untersuchung vorhanden ist, und rationaler Konstruktionsstrategie, die auf einem bestehenden Kenntnisstand aufbaut (ebd.: $36 \mathrm{f}$.). Je nach Ansatz und bereits vorliegenden Erkenntnissen werden bezüglich der Gesamtfragestellung bzw. -hypothese zu testende Einzelaspekte herausgelöst (u. U. können auch pro Aspekt Teilhypothesen gebildet werden), systematisiert und deren mögliche Ausprägungen expliziert (vgl. bspw. Steiner \& Benesch 2018: 50; Kühne 2013: 24). Hieran schließt sich dann die konkrete Frageformulierung für jeden Einzelaspekt an.

Prinzipiell unterscheidet man bei standardisierten Befragungen zwischen (1) geschlossenen Fragen, bei denen das Spektrum möglicher Antworten bekannt ist, (2) hybriden Fragen, die gegebene Antwortoptionen vorgeben und offene Optionen vorhalten, sowie (3) offenen Fragen, die frei formulierte Antworten ermöglichen (vgl. Reinecke 2014: 604). Eine hohe Anzahl geschlossener Fragen ermöglicht eine stärkere Standardisierung und damit ein besseres Vergleichen der Antworten und ist zudem weitaus effizienter hinsichtlich des Auswertungsaufwandes als eine hohe Anzahl offener Antworten. Jedoch haben offene Fragen den Vorteil, das Teilnehmende Antworten unabhängig von Antwortkategorien in eigenen Worten und damit mit eigenen Gewichtungen, Formulierungen und Betonungen versehen können. Offene Antworten sind daher jedoch hinsichtlich der beinhalteten Informationen stark von der „Verbalisierungsfähigkeit der Befragungsperson" (Porst 2014: 57) und der Genauigkeit deren Wiedergabe als selbstverfasste oder protokollierte Äußerung (ebd.) abhängig.

Reinecke (2014) unterscheidet grundsätzlich in fünf Typen von Fragen hinsichtlich deren „Informationswunsch“: (1) Einstellungen, (2) Fakten/Wissen, (3) Ereignisse/Verhalten/Intentionen, (4) sozialstatistische Fragen, sowie (5) Netzwerkfragen. Diese Kategorien prägen die Wahl der Fragenart:

(1) Einstellungen und Meinungen zu bestimmten Themen können mit mehrstufigen Antwortskalen im „stimme zu/stimme nicht zu“-System erfasst werden. Eine Beispielaussage, zu der dann die Einstellung abgefragt werden würde, kann hier sein: „In meiner Heimatregion gibt es eine zu geringe Vielfalt an Ausbildungsplätzen!“ (mehrstufiges Antwortspektrum zwischen den Polen „stimme zu“ und „stimme nicht zu“)

(2) Fragen zu Fakten/Wissen beinhalten oftmals konkrete und distinkte Antwortoptionen, die bspw. im Single-Choice oder Multiple-ChoiceStil abgefragt werden. Ein Beispiel kann hier sein: „In welcher Branche gibt es in Ihrem Heimatort Ausbildungsplätze?" (Antworten könnten eine Auswahl von Branchen vorgeben, bspw. Pflegeberufe, etc.)

(3) Bei Ereignis- bzw. Verhaltensfragen unterscheidet Reinecke (2014: 606f.) zwischen Fragen, die die Prävalenz von Ereignissen messen (bspw. „Werden an Ihrer Schule Informationsveranstaltungen zum regionalen Arbeitsmarkt durchgeführt?"), und Fragen, welche die Inzidenz von Verhalten messen (bspw. „Wie oft haben Sie in der Schule an Informationsveranstaltungen zum regionalen Arbeitsmarkt teilgenommen?").

(4) Sozialstatistische Fragen beziehen sich auf Antwortende und können bspw. demographische Daten, Einkommen, Alter oder Geschlecht abfragen.

(5) Netzwerkfragen können das „Beziehungsnetz“ der Antwortenden erheben (Reinecke 2014: 608) und ermöglichen die Rekonstruktion sozialer Netzwerke. Ein Beispiel wäre: „Mit wem diskutieren Sie die Frage, ob Sie zukünftig wegziehen oder in Ihrer Heimatregion verbleiben wollen?"

Insbesondere bei einstellungs-, fakten- und verhaltensbezogenen Fragen muss grundsätzlich entschieden werden, welche Art von Skala bei Antworten $\mathrm{zu}$ verwenden ist (vgl. Porst 2014: 71; Hollenberg 2016: $14 \mathrm{ff}$.): Nominalskalierte Antworten schließen sich gegenseitig aus, bspw. bei der Angabe von Geschlecht, höchstem Bildungsabschluss (=polytome Skala), Ja/Nein-Entscheidungen (=dichotome Skala) oder der Frage nach einer Wahlabsicht (Porst 2014: 73). Ordinalskalierte Antworten stehen in einer Rangordnung, wobei eine "Gleichabständigkeit“ zwischen den Antworten nicht unbedingt gegeben ist (Porst 2014: 74). Anwendungsbeispiele betreffen Aussagen über Häufigkeiten, Interessen, Zustimmung etc. in Form sog. Ratingskalen (vgl. Steiner \& Benesch 2018: 58) oder semantischer Differenziale (vgl. Porst 2014: 95 ff.). Intervallskalierte Antworten unterscheiden sich hiervon insofern, als dass gleiche Intervallabstände gegeben sind (insbes. Porst 2014: 75 f.; weiterführend zur Umsetzung im Rahmen sozialwissenschaftlicher Fragestellungen Porst 2014: 77 ff.; Callegaro 2015: 66 ff.).

Weiterhin sind Fehlerquellen zu berücksichtigen (vgl. Meier Kruker \& Rauh 2005: 100), die in be- 
grenztem Maße durch eine sorgsame Konzeption umgangen werden können:

- Im Fragebogen selbst können bereits Ungenauigkeiten oder Voreingenommenheiten (vgl. Bogner \& Landrock 2015: 7 ff.) in der Frageformulierung bzw. in der Frageplatzierung (sog. „Halo-Effekt") vorkommen. Auch kann es sein, dass Antwortkategorien spezifische Annahmen bereits implizieren oder nicht vollständig sind. Eine kritische Arbeit am Fragebogenentwurf entsprechend Schritt 4 (Tests und Revisionen; s. u.) kann hierbei Abhilfe schaffen.

- Auf Seiten der Antwortenden sind einige Verzerrungen möglich (vgl. Bogner \& Landrock 2015: $2 \mathrm{ff}$.), die nur bedingt beeinflussbar sind: Einerseits könnten vorrangig sozial erwünschte Antworten gegeben werden, Antworten generell verweigert werden oder eine gewisse Gleichgültigkeit sich zwar in getätigten, aber nicht bewusst gewählten und reflektierten Antworten niederschlagen. Diesen Phänomenen ist nur bedingt beizukommen, ggf. durch eine besonders zielgruppenorientierte Ansprache und Motivation im Vornherein oder im Einleitungsteil. Andererseits sind auch schematische Antwortmuster (bspw. immer Antwort a), ein Hang zu Antwortkategorien die keine Extrema sind oder ein Hang zu „Weiß Nicht“-Kategorien möglich. Diesen Fällen kann im Rahmen der Frage- und Antwortkonzeption begegnet werden, indem bspw. Fragearten oder Antwortskalen häufig gewechselt, bzw. „Weiß-Nicht“-Kategorien oder neutrale Kategorien bewusst und sparsam eingesetzt werden.

- Auf Seiten der Interviewenden bzw. der Erhebungssituation sind zudem einige Fehlerquellen zu verorten (vgl. Bogner \& Landrock 2015: 5 ff.; Glantz \& Michael 2014): Wenn die Antwortenden im Beisein einer interviewenden Person den Fragebogen ausfüllen, bzw. ein Ausfüllen im Beisein Dritter (bspw. Angehöriger) geschieht, können subtile Beeinflussungen stattfinden, so dass im engeren Sinn keine Einzelmeinung erhoben werden würde. Auch kann es vorkommen, dass bei einer prominenten Nennung von Firmen oder Förderinstitutionen ggf. die Antworten von der Meinung der antwortenden Person diesen Institutionen gegenüber beeinflusst werden. Nicht zuletzt ist auch ein wissenschaftsethisch verwerfliches und teils kriminelles Fälschen von Erhebungsdaten durch Forschende ein unglückliches aber durchaus vorkommendes Phänomen (vgl. Blasius 2014), dem durch ein kritisches und konstruktives Arbeiten im Konzeptionsteam, d. h. den an der Konzeption des Fragebogens Beteiligten, begegnet werden kann.

\section{Schritt 4: (Pre-)Tests und Revisionen der Frage- bogenentwürfe}

Nach der grundlegenden Konzeption des Fragebogens sollte dieser nicht einfach zirkuliert werden; vielmehr werden (i) Ungenauigkeiten bei der Frageformulierung, (ii) spezifische Verzerrungen oder (iii) unverständliche Anweisungen häufig erst bemerkt, wenn der Fragebogen tatsächlich von Personen benutzt wurde, die nicht an der Erstellung beteiligt waren. Insofern empfehlen wir auf der Basis unserer Erfahrungen zumindest drei Test- und Revisionsschritte - wobei im Prinzip auch andere (Pre-)Testverfahren möglich sind (vgl. Porst 2014: 189 ff.). Diese Kaskade an Revisionen zielt darauf ab, den Fragebogenentwurf graduell aus unterschiedlichen Perspektiven auf dessen Validität und Verständlichkeit hin zu verbessern:

Erstens sollte der erste Fragenbogenentwurf V1 durch das Konzeptionsteam intern beantwortet werden. Der Fokus muss hier einerseits auf das Offenlegen von fehlenden Fragen und möglichen Verzerrungen liegen und gleichzeitig antizipieren, ob die vorgegebenen Antwortmöglichkeiten bei geschlossenen Fragen das Spektrum aller möglichen Antworten nahezu komplett abdecken. Ist dies nicht der Fall, könnten sonst offene Fragen bzw. offene Antwortfelder zu häufig notwendig sein, was den Auswertungsaufwand erhöht. Zudem ist zu prüfen, inwieweit die Fragen insofern zu spezifisch sind, als dass ggf. problematische Deanonymisierungen von Antwortenden erfolgen können (vgl. Hammer \& Knopp 2015). Alle gefundenen Auffälligkeiten sollten in der Gruppe diskutiert und im Ergebnis in den Entwurf V2 eingearbeitet werden.

Zweitens muss der resultierende Fragebogenentwurf V2 nun erneut nach den Änderungen auf neu entstandene unlogische Frageplatzierungen, doppelte Fragen oder andere Fehler überprüft werden, wobei ein komplettes Neubeantworten durch das Konzeptionsteam i.d.R. nicht notwendig ist. Ziel ist es vielmehr, zu prüfen, inwieweit die Änderungen an Entwurf V1 neue Fehler hervorgebracht haben. Das Ergebnis dieses Schrittes ist der Fragebogenentwurf V3.

Drittens kann nun der Fragebogenentwurf V3 im Rahmen eines sog. Pre-Tests (Reinecke 2014: $614 \mathrm{f}$;; vgl. Weichbold 2014) an eine eng umgrenzte Stichprobe von Teilnehmenden verteilt werden - die jedoch zwingend nicht dem Konzeptionsteam angehören. Ziel ist es, durch das Testen der Befragungen bei externen Personen einerseits ein realistisches Bild der Adäquanz der Frageformulierung und Fragebogenlogik, mögliche Verzerrungen sowie einen Eindruck hinsichtlich der Verständlichkeit des Fragebogens zu 
erhalten. Zudem kann im Anschluss hieran ggf. eine Überarbeitung von Antwortkategorien erfolgen. Alle Auffälligkeiten sollten in der Konzeptionsgruppe diskutiert und dann ggf. eingearbeitet werden. Alternativ oder komplementär dazu sind im Sinne eines MixedMethods-Ansatzes (vgl. Creswell \& Plano Clark 2007: 76) mit den Pre-Test-Teilnehmenden individuell oder in Gruppen Feedbackgespräche zur inhaltlichen Gestaltung des Fragebogens denkbar, deren Ergebnisse systematisch ausgewertet und hinsichtlich ihrer Konsequenzen für die Fragebogenrevision dokumentiert werden sollten. Das Ergebnis dieser möglichen Revisionsschritte ist der finale Fragebogen.

\section{Schritt 5: Durchführung der Online-Befragung}

In der Folge kann der finale Fragebogen mittels eines webbasierten Werkzeugs digital umgesetzt werden, wobei hier nach Fertigstellung eine sorgfältige Kontrolle folgender Aspekte notwendig ist; ggf. sind dazu weitere technische Testläufe einzuplanen:

- Vollständigkeit

- Rechtschreibung und Formatierung

- Logische Folge und Verknüpfungen bei Nutzung von Filterfragen

- Zugriffsmöglichkeiten (via permanenter URL) und deren zeitliche Beschränkungen.

Die Zirkulation des Zuganges zum Fragebogen erfolgt i.d.R. per Einbettung der URL in eine E-Mail oder ein anderes Textdokument, über das die Teilnehmenden Zugriff auf den Fragebogen haben (vgl. Callegaro et al. 2015: $115 \mathrm{ff}$.). Ein kommunizierter Zeitraum für die Durchführung ist empfehlenswert, woraufhin der Fragebogen dann depubliziert werden sollte, um kontinuierliche Dateneinträge zu verhindern.

Wagner und Hering (2014: 666f.) betonen, dass trotz der scheinbaren Einfachheit von Online-Befragungen potentielle Teilnehmende einige motivationsund kompetenzbezogene Hürden nehmen müssen, bevor tatsächlich eine verwertbare Antwort entsteht. Insofern müssen Fälle des kompletten Verweigerns der Teilnahme (genannt „Unit-Nonresponse“), einzelner nicht beantworteter Fragen (genannt „Item-Nonresponse") oder des Abbrechens der Befragung (genannt „Drop-Out“) vermieden werden (ebd.). Die komplette Teilnahmeverweigerung ist durch eine ansprechende und motivierende Ausgestaltung des Fragebogens und dessen erklärenden Einleitungsteil in begrenztem Maße adressierbar. Eine überschaubare Länge und Bearbeitungsdauer kann hierbei, genauso wie bei der Verhinderung von Drop-Outs, positive Effekte haben. Hinsichtlich der Item-Nonresponse-Fälle empfiehlt Porst (2014: 130) eine kritische Auswertung solcher Fälle im Rahmen von Pre-Tests (vgl. Schritt 4), jedoch auch eine Überprüfung womöglich falscher Frageformulierung oder falsch gesetzter Filterfragen (ebd.). Auch die Wahl der Antwortkategorien, bspw. das Weglassen von neutralen Antwortkategorien bei Ratingskalen kann zu einer fragespezifischen Antwortverweigerung führen (vgl. ebd.: 84). ${ }^{3}$

\section{Schritt 6: Auswertung der Online-Befragung}

Während bei Offline-Fragebögen die Antworten vor der Auswertung in eine Auswertungssoftware (bspw. Excel, SPSS, PSPP) manuell eingepflegt werden müssen, ist dieser Schritt bei Online-Fragebögen i.d.R. semiautomatisch über gängige Export-/Import-Verfahren zeiteffizient möglich. Einer grundlegenden Sicherung der Befragungsergebnisse im Rohzustand ist jedoch ebenso besondere Bedeutung beizumessen, wie einer Datenbereinigung (vgl. Wagner \& Hering 2014: 668), sowie der Dokumentation in Form der Rohversionen des Fragebogens, Variablenlisten, usw.

Hinsichtlich des Schrittes der Fragebogenauswertung unterscheiden Kirchoff et al. (2010: $47 \mathrm{ff}$.) eine Grundauswertung, sowie weiterführende explorative Verfahren:

Eine Grundauswertung betrifft die fragenspezifische Aggregation aller Einzelantworten, ggf. aufbereitet in Prozentangaben (ebd.: 49), sowie die Aggregation von Antwortkategorien (ebd.: 52) oder durch Klassifikationen (ebd.: 53). Ziel ist hierbei eine datennahe Berichterstattung über die Ergebnisse der Befragung, ggf. unter Hinzuziehung deskriptiver statistischer Verfahren und graphischer Visualisierungen (ebd.: 59 ff.; Hollenberg 2016: 31 ff.).

Eine weiterführende explorative Auswertung hat hingegen das Ziel, komplexe Strukturen und Besonderheiten in den Daten offenzulegen. Gängige Verfahren diesbezüglich sind bspw.

- Clusteranalysen (d.h. die Gruppierung von Ausprägung auf der Basis von Ähnlichkeiten oder Distanzen; vgl. Kirchhoff 2010: 75),

- Faktorenanalysen (d. h. verschiedene Verfahren zur mathematischen Reduktion einer Vielzahl von Variablen auf eine kleinere Menge hypothetischer Variablen), und

- Korrespondenzanalysen für kategoriale Daten.

Welches Verfahren hierbei am besten geeignet ist, hängt stark von der Fragestellung der Befragung,

\footnotetext{
3 Gleichzeitig kann das Nutzen einer neutralen Kategorie sich ebenfalls negativ auf das Antwortverhalten bestimmter befragter Personen auswirken (Steiner \& Benesch 2018: 60). Steiner und Benesch, eingedenk dieser paradoxen Rolle von neutralen Kategorien in ihrer Wirkung auf mögliche Antwortverweigerungen und -verzerrungen, plädieren deswegen dafür, diese Art von Antwortkategorien inhaltlich und auf die Fragestellung und Zielgruppe abgestimmt bewusst einzusetzen (ebd.).
} 
der Stichprobe, den zugrundeliegenden Hypothesen und den Rahmenbedingungen $a b$, um nachfolgend geeignete Interpretationen der Daten vornehmen zu können. An dieser Stelle sei deswegen auf einschlägige Einführungen in die Datenanalyse im Kontext von Fragebogenerhebungen verwiesen (bspw. Porst 2014; Baur \& Blasius 2014).

\section{Fragebögen: analog vs. online}

Neben diesen konzeptionellen Schritten stellt sich zudem abschließend die Frage, ob bei der Verwendung eines Fragebogens auf konventionelle Mittel in Form gedruckter Dokumente zurückgegriffen wird oder auf einen Online-Fragebogen. Bei der Durchführung von Online-Befragungen in der empirischen Sozialforschung werden in der Literatur einige Nachteile und Herausforderungen genannt (vgl. Taddicken 2013: 208; Evans \& Mathur 2018; Nayak \& Narayan 2019):

- Wahrgenommene Anonymität beim Versenden bzw. Empfangen der Umfrage

- Teils sehr geringe Rücklaufquoten und damit verbunden

- Schwierigkeiten bei der Steuerung der Stichprobenzusammensetzung

- Online-Fragebögen werden als unpersönlich empfunden

- Keine Interventionsmöglichkeiten bei Verständnisfragen der Rezipienten

- Herausforderungen beim (technischen) Sicherstellen der Privatsphäre

- Wenig Kontrolle über die Befragungssituation

Demgegenüber stehen aber zahlreiche Vorteile, die diesen Problemen gegenüber teils erheblich überwiegen (ebd.):

- Potentiell sehr große Reichweite und daher

- Potentiell sehr große Stichproben

- Flexibilität beim visuellen Design, der Verteilung an potentielle Adressat*innen und bei der Auswertung angesichts leichteren Datenexports

- Automatisierung der Präsentation der Erstergebnisse in Echtzeit und daher

- Deutlich einfachere Dateneingabe und -analyse

- Einfache Umsetzung von Pfadabhängigkeiten unterschiedlicher Antworten

- Kosteneffizienz

- Vergleichsweise geringer zeitlicher Erhebungsaufwand

Vor allem auch im schulischen Kontext stellt sich bei der Integration in einen forschenden Unterricht die Frage, ob Online(!)-Fragebogen oder nicht? Diese Wahl kann arbeitsökonomisch begründet werden und wird durch die konkrete Ausgestaltung des Lehrplans, die zur Verfügung stehende Zeit und das Budget beeinflusst. Gerade wegen der eben gelisteten Vorteile (Flexibilität, Automatisierung, Echtzeit-Resultate) bieten sich Online-Fragebögen an, um im Unterricht entweder selbst thematisiert oder als Werkzeug eingesetzt zu werden. Ein Beispiel hierfür werden wir im folgenden Kapitel vorstellen.

\section{Online-Fragebögen im GW-Unterricht: ein Unterrichtsbeispiel}

Im Folgenden wollen wir uns nun der Frage widmen, wie das bisher Genannte im GW-Unterricht adaptiert werden kann und entsprechend werden wir eine Möglichkeit diskutieren, das Thema der Wegzugsmotivation Jugendlicher mithilfe eines Online-Fragebogens im Unterricht zu bearbeiten.

Hier bieten sich inhaltlich (Wegzugsmotivationen Jugendlicher in der eigenen Lebenswelt und damit einhergehend einerseits die biographische Mobilität Jugendlicher und andererseits die Bedeutung ländlicher und urbaner Regionen bzw. deren Konstruktion) zahlreiche Anknüpfungspunkte an. Entsprechende Referenzdokumente liefern hier diverse Andockmöglichkeiten. In den deutschen Bildungsstandards im Fach Geographie (DGfG 2020) trifft dies im Kompetenzbereich Fachwissen insbesondere auf die Fähigkeiten F3 (Fähigkeit, Räume unterschiedlicher Art und Größe als humangeographische Systeme zu erfassen) und F5 (Fähigkeit, individuelle Räume unterschiedlicher Art und Größe unter bestimmten Fragestellungen zu analysieren) zu. Im österreichischen GW-Lehrplan für die Sekundarstufe 2 (Hinsch et al. 2014) finden sich Anknüpfungspunkte in den Semestern 6 (im Bereich „Demographische Entwicklung und gesellschaftspolitische Implikationen beurteilen") und 8 (im Bereich „Städte als Lebensräume und ökonomische Zentren untersuchen"). Darüber hinaus werden direkt mehrere Basiskonzepte des Lehrplans adressiert, womit die inhaltliche Angemessenheit des Gegenstands mehr als gegeben ist:

- Raumkonstruktion und Raumkonzepte (über die subjektive(n) Bedeutung(en) der Weg- und Zuzugsorte sowie deren Reflexion)

- Regionalisierung und Zonierung (über die Einbettung des Gegenstands in regionale und überregionale Kontexte)

- Diversität und Disparität (über die Thematisierung unterschiedlicher Lebensrealitäten im Kontext potenzieller Wegzüge)

- Wahrnehmung und Darstellung (über die Auseinandersetzung mit subjektiv wahrgenommenen und aktiv kommunizierten Push- oder Pull-Faktoren unterschiedlicher Regionen) 
- Nachhaltigkeit und Lebensqualität (über die subjektiv lebensweltliche Anbindung der Auseinandersetzung mit eigenen Wegzugsmotiven)

- und letztlich natürlich Kontingenz (über die sich zwangsläufig ergebende Erkenntnis, dass der Gegenstand differenziert betrachtet werden muss)

Neben dieser inhaltlichen Begründung lassen sich aber auch kompetenzorientierte Argumente für die Thematisierung dieses Gegenstands identifizieren. Sofern man dieses Thema nicht narrativ-exemplarisch (z. B. mithilfe des Schulbuchs), sondern proaktiv eben beispielsweise mit einem (Online-)Fragebogen forschend - thematisiert, werden methodisch und damit v.a. im Hinblick auf die Kompetenzorientierung im Geographie-/GW-Unterricht (Kanwischer 2011; Padberg 2012; Pichler 2013) bei der Bearbeitung des Themas die Kompetenzbereiche Erkenntnisgewinnung/Methoden, Kommunikation und Beurteilung/ Bewertung (und je nach Adaption auch Handlung) (DGfG 2020) bzw. die Bildungsbereiche Sprache und Kommunikation, sowie Mensch und Gesellschaft (BMB 2016) direkt adressiert. Darüber hinaus und jenseits explizit geographischer Bildungsanliegen wird so zudem problemorientiertes Lernen (Reusser 2005) und forschendes Lernen (Tillmann 2015) gefördert. Speziell im Hinblick auf die Integration eines Online(!)-Fragebogens in den Unterricht werden zusätzlich Unterrichtsprinzipien wie Medienbildung (BMBF 2014) und v.a. die in Österreich inzwischen erlassene verbindliche Übung Digitale Grundbildung (BMBWF 2018) angesprochen.

Bevor wir im Weiteren einen konkreten Unterrichtsvorschlag liefern, muss zunächst darauf aufmerksam gemacht werden, welche mögliche Spannweite sich bei der Integration des Werkzeugs Online-Fragebogen in den Unterricht eröffnet. Hier können inhaltlich und strukturell mehrere Komplexitätsstufen identifiziert werden, so z. B.:

- von aktiv (Schüler*innen konzipieren den Fragebogen selbst, streuen ihn selbst, analysieren die Ergebnisse selbst) bis passiv (die Lehrperson stellt den Fragebogen zur Verfügung und diskutiert dessen Ergebnisse im Unterricht),

- von forschend (Schüler*innen beforschen eine Zielgruppe) bis beforscht (Schüler*innen sind die direkten Adressat*innen des Fragebogens),

- von offen (der Fragebogen wird jenseits des Regelunterrichts gestreut) über semi-offen (der Fragebogen wird bspw. nur innerhalb der Schule gestreut) bis quasi geschlossen-exemplarisch (der Fragebogen wird nur im Klassenverband beantwortet),

- von schuljahrbegleitender Erstellung im Rahmen des GW-Unterrichts, über mögliche reduzierte
Erstellungszeiträume in Projektwochen, bis hin zu kompakten Nutzungen in Einzelstunden mit eingeschränktem Erarbeitungsumfang.

Diese (nicht vollständige) Skalenliste zeigt bereits enorme Variationsmöglichkeiten (auch in Kombination der einzelnen Kategorien untereinander). Hierbei wird offensichtlich, dass langfristige und projektorientierte Settings insofern umso erforderlicher werden, je aktiver, explizit forschender und offener der Fragebogen im Unterricht integriert wird, da somit die Komplexität und der Vorbereitungs- und Bearbeitungsaufwand entsprechend größer werden. Dies wiederum ist im Regelunterricht selten möglich, daher haben wir uns im folgenden Vorschlag bewusst für ein kleineres Setting entschieden, welches jederzeit auch problemlos im alltäglichen Regelunterricht umgesetzt werden kann. Zudem kommen dann insbesondere die expliziten Vorteile von Online(!)-Fragebögen (in Form schneller und transparenter Umsetzbarkeit, Datensammlung und Ergebnispräsentation in Echtzeit vgl. Kap. 3) umso mehr zum Tragen, und erleichtern dementsprechend eine Anwendung im Unterricht, die sich beispielsweise mit einem analogen Fragebogen ungleich schwerer gestalten ließe.

Die Nutzung eines Online-Fragebogens im Unterricht verschiebt sich dementsprechend in unserem Beispiel weg vom Erstellungsprozess (der vorab skizzenhaft thematisiert werden kann) hin zur Praxis der Durchführung und Auswertung im Unterricht. Das inhaltliche Ziel des folgenden Unterrichtsbeispiels ist daher die Erhebung von (potentiellen) Wegzugsmotivationen von Jugendlichen im Kontext des eigenen Lebensumfelds (i. e. des Ortes der eigenen Schule), wobei sich die Schüler*innen selbst als Stichprobe verstehen. Die didaktische Zielstellung ist dann im Weiteren, dass Schüler*innen in die Lage versetzt werden, aus dieser Erhebung Rückschlüsse (oder deren Unmöglichkeit) auf die Gesamtsituation zu diskutieren und Folgen für den eigenen (Wohn)Ort zu thematisieren. Um eine entsprechende Schüler*innenorientierung im Kontext des Lebensweltbezugs (vgl. Pichler et al. 2017) herstellen zu können, verorten wir diese Einheit in der Sekundarstufe II, idealerweise im Abschlussjahrgang, da hier das Thema für die Schüler*innen am relevantesten ist (weil sie sich selbst in absehbarer Zukunft direkt mit dieser Frage beschäftigen werden müssen).

Die nun folgende Planung über zwei Doppeleinheiten ist dabei bewusst offen gestaltet, um thematisch (z. B. Adaption in einem nicht ländlich geprägten Schulumfeld und entsprechender Justierung der Fragestellung) und organisatorisch (z. B. Reduktion auf weniger Unterrichtseinheiten durch Auslassung und/oder Anpassung einzelner Schritte) größtmögliche Variationsmöglichkeiten sicherstellen zu können. 
Je nachdem, wie umfangreich und komplex die Sequenz dann tatsächlich umgesetzt wird und wie aktiv Schüler*innen in die einzelnen Teilschritte involviert werden, adressiert die Planung im einfachsten Falle ein praktisches- und im umfangreichsten Falle ein emanzipatorisches Vermittlungsinteresse (Vielhaber 1999).

Das konkret benannte Groblernziel der Unterrichtssequenz ist somit, dass Schüler*innen über die von der Lehrperson unterstützte und transparent gemachte Erhebung und Auswertung von Wegzugsmotivationen Jugendlicher anhand einer exemplarischen Stichprobe (= der eigenen Klasse) Rückschlüsse für die demographische Regionalentwicklung des eigenen Umfelds ziehen, diese interpretieren und diskutieren, sowie potentielle Folgen abschätzen. Entsprechende Feinziele sind hier bewusst nicht formuliert, da sich diese je nach konkreter Variation teils erheblich unterscheiden können.

Mögliche Variationsvorschläge werden jeweils am Ende einzelner Zwischenschritte genannt. Der für die Lernumgebung zu erstellende und auszufüllende Kurzfragebogen wird abhängig von der jeweiligen Klassendynamik flexibel formuliert. Dabei spielt die Lehrperson moderierend aber auch intervenierend eine zentrale Rolle. Daher ist die vorherige Auseinandersetzung der in Kapitel 2 gelisteten Richtlinien und Kriterien durch die Lehrkraft essentiell, um ggf. im Unterrichtsgeschehen angemessen (falls nötig, intervenierend) reagieren zu können.

\subsection{Erste Unterrichtseinheit}

\section{Einstieg}

Als Einstieg zur Unterrichtseinheit schlagen wir die Präsentation eines (Online-)Zeitungsartikels vor, der sich lose um das Mobilitätsverhalten von Jugendlichen dreht. Dies kann makroperspektivisch z. B. ein genereller Artikel zu Landflucht von Jugendlichen $\operatorname{sein}^{4}$ oder ein Artikel auf einem etwas kleinerem Maßstab zur Bevölkerungsentwicklung im eigenen lokalen oder regionalen Umfeld, der den Wegzug junger Menschen thematisiert. Dabei geht es nicht darum, jenen Artikel zu analysieren. Eine bloße Präsentation des Titels und Teasers via Beamer genügt völlig, um damit das Thema als Diskussionsimpuls und Denkanstoß zunächst in den Raum zu stellen.

Im nächsten Schritt wird der Artikel in Bezug zum eigenen Umfeld gesetzt und die Frage gestellt, wie sich dieses Phänomen in der eigenen Gemeinde bzw. Stadt zeigt. Konkrete Fragebeispiele können sein:

z. B. https://www.spiegel.de/wirtschaft/soziales/deutschland-die-extreme-landflucht-der-jungen-und-ihre-gruende-a-1292981.html
- „Was glaubt Ihr, wie ist das Abwanderungsverhalten Jugendlicher in unserer Gemeinde/Stadt charakterisiert?"

- „Habt Ihr vor, nach dem Schulabschluss abzuwandern?"

- „Warum/warum nicht?“

- „Wenn ja, wohin?“

- „Was bedeutet das kurz-/mittel-/langfristig für unsere Gemeinde/Stadt?"“

In einem Unterrichtsgespräch werden Wortmeldungen gesammelt und durch die Lehrperson moderiert, um die Klasse für dieses Thema zu sensibilisieren und die eigene Betroffenheit zu adressieren.

Variationsmöglichkeiten: Je nach konkretem Kontext im eigenen Umfeld können diese Fragen thematisch variiert werden.

\section{Überleitung}

Nach diesem kurzen Einstieg erfolgt nun die Überleitung durch die Lehrperson, dass dieses eben im Gespräch sehr lose erhobene Stimmungsbild nicht geeignet ist, um die Frage nach der Situation vor Ort hinreichend zu beantworten. Der Aspekt der Repräsentativität als ein Gütekriterium von Befragungen kann hierbei flankierend eingeführt werden. In einem Unterrichtsgespräch wird nun skizziert, dass hier eine strukturiertere Befragung notwendig ist und auf das Werkzeug eines Fragebogens verwiesen, mit dem differenziert erhoben werden kann, ob und v. a. wie viele Jugendliche Wegzugsmotive haben, wenn ja, welche und ob sich diese je nach Gruppierung, Region, Alter oder Schulform weiter unterscheiden. Wichtig ist hier, dass die Lehrkraft dabei auf die in Kapitel 2 diskutierten Möglichkeiten und Grenzen der Erhebung (bzw. des daraus folgenden Erkenntnisgewinns) eingeht.

In einem nächsten Schritt wird nun die Frage der Operationalisierung in den Raum gestellt, i.e. welche Fragen wären hierbei essenziell, wichtig oder interessant und wem müsste man diese Fragen wie stellen? Nach einer kurzen Diskussion wird nun zur ersten Erarbeitungsphase übergeleitet.

\section{Erarbeitung - Gruppenarbeit}

Hierfür werden nun Kleingruppen gebildet und die Schüler*innen erhalten den Arbeitsauftrag, in jenen Kleingruppen geeignete Fragen für einen solchen Fragebogen zu erarbeiten, zu diskutieren und festzuhalten. Um möglichst nahe an potentiellen Präkonzepten der Schüler*innen zu bleiben, ist dieser Arbeitsauftrag bewusst offen gehalten, um eventuelle Verzerrungen oder Beeinflussungen zunächst zu vermeiden. Entsprechend wichtig ist dann der nächste Schritt der 
moderierten und ggf. visualisierenden (Zwischen)Sicherung.

Variationsmöglichkeiten: Hier besteht je nach gewählter inhaltlicher Ausrichtung bzw. geplanter Ausdifferenzierung des Fragebogens die Möglichkeit, vorab bereits Sub-Themen für die einzelnen Gruppen festzulegen.

\section{(Zwischen)Sicherung}

Im Anschluss werden die von den einzelnen Gruppen erarbeiteten Fragen im Plenum vorgestellt, durch die Lehrperson (an einer Pinnwand oder auf einem Whiteboard) gesammelt und thematisch und/oder strukturell geclustert. Im nun folgenden Unterrichtsgespräch werden diese Fragen dann zusammengefasst (bei mehreren inhaltlich ähnlichen oder gleichen Fragen), adaptiert (bei unpräzisen Fragen oder bei Fragetypen, die für die Erhebung des zu Erfragenden eher ungeeignet sind) und ergänzt (bei wichtigen inhaltlichen Aspekten, die evtl. nicht berücksichtigt wurden). Hierbei sollte die Lehrkraft auf die in Kapitel 2 beschriebenen Kriterien achten und ggf. - in reduzierter Form - im Gespräch darauf verweisen. Hierfür ist ein gewisser Vorbereitungsaufwand nötig, um flexibel reagieren zu können. Eine exemplarische Sammlung möglicher Fragen für einen Beispiel-Fragebogen findet sich zur Illustration im Anhang (vgl. Anhang A). Je nach konkretem Setting können hier zusätzliche Anmerkungen von Schüler*innen berücksichtigt werden. Abschließend wird der so entstandene Fragenkatalog gesichert (Export/Screenshot im Falle des Whiteboards oder Fotographie im Falle der Pinnwand) und den Schüler*innen (im Falle des Vorhandenseins eines digitalen Learning Management Systems) zur Verfügung gestellt und auf die nächste Unterrichtseinheit verwiesen.

Variationsmöglichkeiten: Je nachdem, wie viel Zeit für die gesamte Einheit eingeräumt wird, kann hier die aktive Rolle der Schüler*innen erhöht werden (beim Zusammentragen, Clustern, Ergänzen des Fragenpools) und die Lehrkraft nur im Hintergrund unterstützend agieren.

\subsection{Zwischenschritt}

Zwischen der ersten und der zweiten Unterrichtseinheit werden die Fragen durch die Lehrperson digitalisiert und in einen Online-Fragebogen übertragen. Durch die (im Vergleich zu wissenschaftlich validen Fragebögen) relativ einfache Struktur gibt es hierfür zahlreiche kostenfreie und einfach zu bedienende Werkzeuge und Plattformen, z. B. Google Formulare, SurveyMonkey oder Microsoft Forms (im Education Bundle von Microsoft), für die es ebenso einfache und gut nachvollziehbare Anleitungen gibt. ${ }^{5}$ Entsprechend ist das Erstellen und Veröffentlichen eines Fragebogens an sich mit minimalem Aufwand verbunden, der die normale Unterrichtsvorbereitung kaum übersteigt.

Variationsmöglichkeiten: Hier besteht natürlich auch die Möglichkeit, die Erstellung des Fragebogens oder Teile davon durch Schüler*innen vornehmen zu lassen.

\subsection{Zweite Unterrichtseinheit}

\section{Einstieg}

Bezugnehmend auf die vergangene Unterrichtseinheit wird durch die Lehrperson der inhaltliche Anschluss an die Erarbeitung der Fragen hergestellt und darauf verwiesen, dass der im Zwischenschritt erstellte Fragebogen nun im Klassenverband ausprobiert wird. Entsprechend wird der Zugangslink zum Fragebogen durch die Lehrperson (mittels Kurz-URL oder bspw. QR-Code) zur Verfügung gestellt und zum Einstieg durch die Schüler*innen ausgefüllt. Hierfür können die Schüler*innen die eigenen Smartphones verwenden oder - je nach Ausstattung der Schule - vorhandene Schulhardware genutzt werden (z. B. Notebookoder Tablet-Klassen). ${ }^{6}$

Variationsmöglichkeiten: Durch die Ortsungebundenheit eines Online-Fragebogens kann dieser auch außerhalb des eigentlichen Unterrichtsgeschehens ausgefüllt werden. Dies bietet sich z. B. an, wenn die Lernumgebung parallel in mehreren Klassen durchgeführt wird und die Diskussion der Ergebnisse im nächsten Schritt nicht nur auf dem Fragebogenrücklauf dieser einen Klasse beruht.

\section{Erarbeitung}

Im Anschluss an das Ausfüllen des Fragebogens durch die Schüler*innen werden die ersten Ergebnisse via Beamer durch die Lehrkraft präsentiert. Hier zeigt

\footnotetext{
Bestehende Werkzeuge entwickeln sich hier ständig weiter und unterliegen schnelllebigen Veränderungen. Die grundlegende Logik ist jedoch stets gleich: 1) Fragebogen anlegen, 2) Fragen hinzufügen, Fragekategorie festlegen, und ggf. Antwortmöglichkeiten festlegen, 3) Fragebogen prüfen, 4) Fragebogen veröffentlichen, 5) Link/URL zum Fragebogen versenden, 6) Ergebnisse einsehen. Für die entsprechenden Werkzeuge stehen jeweils sehr gut dokumentierte Anleitungen zur Verfügung, so z. B.:

Google Formulare: https://support.google.com/docs/ topic/6063584?hl=de, SurveyMonkey: https://help.surveymonkey.com, MS Forms: https://support.microsoft.com/de-de/forms 6 Auch wenn sich die konkrete Ausstattung an einzelnen Schulen teils erheblich unterscheidet, ist diesbezüglich zeitnah mit einem erheblichen Schub zu rechnen. Hier sei z. B. - in Österreich auf den 8-Punkte-Plan der Initiative „Digitale Schule“ verwiesen: https://digitaleschule.gv.at/
} 
sich der entscheidende pragmatische Mehrwert von Online-Fragebögen für den Unterricht, denn diese Ergebnisse müssen nicht erst aufgearbeitet werden, sondern werden (je nach verwendeter Plattform etwas unterschiedlich) in Echtzeit als Zusammenfassung in der Benutzeroberfläche der jeweiligen Plattform ausgegeben und können somit direkt und unmittelbar betrachtet und in einem Unterrichtsgespräch diskutiert werden (eine exemplarische Erstzusammenfassung findet sich zur Illustration in Anhang B). Mögliche Fragen können sein:

- „Haben wir das so erwartet?“

- „Was überrascht uns hier?“

- „Entspricht dieses Ergebnis gegenwärtigen Trends?"

\section{Vertiefung - Gruppenarbeit}

Im nächsten Schritt werden wieder Kleingruppen gebildet und jede Gruppe bekommt den Arbeitsauftrag, sich basierend auf diesen Ergebnissen mit einer Teilfrage zu beschäftigen, diese zu diskutieren und das Ergebnis festzuhalten. Hier gibt es mindestens zwei Möglichkeiten:

Einerseits können die Ergebnisse differenzierter aufgearbeitet und interpretiert werden. So kann bspw. nach soziodemographischen Merkmalen unterschieden werden und jede Arbeitsgruppe beschäftigt sich mit einer Teilgruppe (z.B. männlich vs. weiblich) oder jede Gruppe beschäftigt sich mit einem Teilaspekt. Hierfür ist allerdings eine Vorfilterung der Ergebnisse nötig. Die meisten Plattformen bieten einen direkten Excel-Export der Ergebnisse an, sodass dieser Filter (in Excel) sehr schnell vorgenommen werden kann. Andererseits kann hier auch inhaltlich differenziert werden, sodass sich jede Teilgruppe mit einer inhaltlichen Frage beschäftigt (z. B. „Was bedeuten diese Ergebnisse für die Regionalentwicklung?“ oder „Was sind noch offene Fragen?").

Variationsmöglichkeiten: Je nach Sozialdynamik der Klasse und methodischem Vorwissen der Schüler*innen kann diese Gruppenarbeitsphase auch ausgelassen und derartige Vertiefungsfragen in Form einer moderierten Diskussion im vorangegangenen Schritt im Plenum bearbeitet werden. Dabei entfällt auch der folgende Schritt 9 und die Ergebnissicherung muss durch die Lehrkraft moderierend sichergestellt werden.

\section{Sicherung}

Abschließend präsentieren die Einzelgruppen ihre Ergebnisse kurz und diese werden von der Lehrkraft zusammengetragen, moderiert und letztlich auch (z. B. in einem LMS) gesichert, sodass sie auch nach der Einheit zur Verfügung stehen. In diesem Unterrichtsgespräch werden die Ergebnisse schließlich auch inhaltlich kontextualisiert, sodass Schlussfolgerungen (z. B. für die demographische Regionalentwicklung) zumindest andiskutiert werden können und sich weitere lohnende Fragen (für bspw. folgenden Unterrichtseinheiten) ergeben (z. B. potentielle lokalpolitische Maßnahmen - je nach Ergebnissen der Umfrage).

Zudem sollte in dieser Diskussion hinsichtlich der bisher gewonnenen Erkenntnisse auch eine Meta-Reflexion initiiert werden („Welche Schlüsse können wir ziehen und welche nicht?“, „Wo müssten wir zusätzliche Fragen stellen?“, „Sind wir als Klasse repräsentativ für unser lokales Umfeld?"). Insbesondere sollte hier auch der Fragebogen als Erhebungsinstrument hinsichtlich seiner Möglichkeiten und Grenzen diskutiert werden („Welche Bereiche hat der Fragebogen abgedeckt und welche nicht?", „Welche Erkenntnisse bedürfen weiterer Bearbeitungsschritte?“, „Wo liegen die Grenzen derartiger Erhebungen?"). Dadurch werden nicht nur die Ergebnisse der Lernumgebung reflektiert; es wird auch generell ein kritischer Blick auf die Erhebung, Präsentation und Interpretation von erhobenen Daten geschult.

Variations-/Fortsetzungsmöglichkeiten: Die Schritte bis hierher können in einem größeren Kontext auch als Pre-Test im Regelunterricht gesehen werden und anschließend in einem projektorientierten Setting in einer größeren Umfrage außerhalb der eigenen Klasse/ Schule münden.

\section{Fazit: Herausforderungen mit und Potentiale von Online-Fragebögen im Unterricht}

Das Zeitalter digitaler Lernumgebungen, OnlineDatenerhebungen und vielfältig verfügbarer Ergebnisdarstellungen erfordert auf Rezipient*innenseite erhöhte Kompetenzen im Umgang mit diesen allgegenwärtigen Mitteln. Die beispielhafte Integration von Online-Fragebögen in den Schulunterricht anhand regionaler Wanderungsdynamiken vermag es darüber hinaus, sowohl inhaltliche als auch methodische Kompetenzen zu schulen. Während die Durchführung und Auswertung von Fragebögen in Form von persönlichen Befragungen im öffentlichen Raum stattfinden könnte, bieten Online-Fragebögen den Vorteil, zeiteffiziente Erhebungen und ad-hoc-Auswertungen bei eingeschränkter Aussagekraft als Beispiele durchzuführen, eben auch direkt im Unterricht.

Für den Einsatz von Online-Fragebögen im Schulunterricht muss jedoch hinsichtlich der in Kapitel 2 diskutierten generellen Vor- und Nachteile weiter differenziert werden. Für die Durchführung von eige- 
nen Forschungsprojekten aus dem Unterricht heraus gelten die o.g. Probleme und Vorteile natürlich gleichermaßen. Wenn - wie im Falle der hier vorgestellten Unterrichtssequenz - das Werkzeug des OnlineFragebogens unterrichtsintern eingesetzt wird, gelten viele dieser Faktoren nur bedingt und es rücken eher pragmatische Fragen in den Vordergrund. Eine offensichtliche Anforderung ist das zwingende Vorhandensein einer stabilen Netzinfrastruktur und entsprechender Endgeräte. Darüber hinaus ist es erforderlich, dass die Lehrperson im Umgang mit entsprechenden Plattformen geübt ist. Diesen - durchaus handhabbaren - Herausforderungen steht wiederum der Vorteil gegenüber, digital gestützt direkt im Unterricht teils komplexe Stimmungsbilder zu erheben, die Ergebnisse in Echtzeit zu visualisieren und diese in den weiteren Unterrichtsverlauf zu integrieren. Dies ist ein aus unterrichtspragmatischer Perspektive nicht zu unterschätzender Mehrwert, denn pointiert gesagt: Dies ist mit analogen Werkzeugen im Unterricht schlichtweg nicht möglich und somit eröffnen sich gänzlich neue Möglichkeiten der interaktiven Unterrichtsstrukturierung.

Ebenso gehen die Potentiale von Online-Fragebögen inhaltlich weit über einfache Abstimmungs- oder Quizwerkzeuge wie bspw. Kahoot! hinaus, welche zwar positive Effekte auf z. B. die Lernmotivation von Schüler*innen haben (vgl. Wang \& Tahir 2020), jedoch ob der ihnen inhärenten geringen Komplexität aus fachdidaktischer Perspektive per se ein sehr begrenztes Potential mit sich bringen. Darüber hinaus kann mit der thematisch offengelegten Auseinandersetzung mit dem Fragebogen selbst der Erkenntnisprozess empirischer Sozialforschung thematisiert und somit auch in der Schule ein Beitrag zum forschenden Lernen geleistet werden. Die hier präsentierte Konzeption, Online-Fragebögen in den Unterricht zu integrieren, stellt dabei nur eine von vielen Variationen dar, um Schüler*innen den Erstellungsprozess, die Durchführung und die Auswertung näher zu bringen bzw. mit der Integration dieses Werkzeugs den Unterricht zu bereichern.

Neben der (zweifelsohne gewinnbringenden, wenngleich auch eher subkutanen und komplexitätsreduzierten) Vermittlung von Logiken empirischer Sozialforschung ist der eigentliche Kern der hier vorgestellten Unterrichtssequenz jedoch ein anderer. Im Rahmen der hier präsentierten Idee dient das (technologiegestützte) Werkzeug des Online-Fragebogens vielmehr als Mittler der ad hoc Erhebung des Stimmungsbilds der Schulklasse. Damit wiederum wird der direkte Lebensweltbezug zum Unterrichtsthema (Wegzug Jugendlicher) hergestellt und ermöglicht so eine weitaus authentischere Auseinandersetzung mit der dahinterstehenden Problemlage. Der Einsatz des
Werkzeugs Online-Fragebogen adressiert hier aus didaktischer Perspektive somit nicht primär die methodischen Kompetenzen, sondern unterstützt die inhaltliche Auseinandersetzung mit einem Thema, indem - wie im beschriebenen Beispiel - in Echtzeit völlig neue Perspektiven auf die sachlich erhobene Lebensrealität der Schüler*innen und den Unterrichtsgegenstand eröffnet werden können. Dies wiederum orientiert sich am beispielsweise im Umgang mit Geoinformation bereits diskutierten Grundsatz (Vogler et al. 2018), dass es nicht darum geht, eine Methode oder Technologie zu lernen, sondern mit(!) der Methode oder Technologie zu lernen.

\section{Danksagung}

Die Veröffentlichung dieses Beitrags wurde vom Open Access Publikationsfonds der TU Dresden unterstützt. Open Access Funding by the Publication Fund of the TU Dresden.

\section{Literatur}

Baur, N. \& J. Blasius (Hrsg.) (2014): Handbuch Methoden der empirischen Sozialforschung. Springer VS, Wiesbaden.

Blasius, J. (2014): Fälschungen von Interviews. In: Baur, N. \& J. Blasius (Hrsg.): Handbuch Methoden der empirischen Sozialforschung. Springer VS, Wiesbaden. S. 323-330.

BMB - Bundesministerium für Bildung (Hg.) (2016): Verordnung der Bundesministerin für Bildung, mit der die Verordnung über die Lehrpläne der allgemein bildenden höheren Schulen geändert wird; Bekanntmachung, mit der die Bekanntmachung der Lehrpläne für den Religionsunterricht an diesen Schulen geändert wird. Wien. BGBl. II Nr. 219/2016 vom 09.08.2016. https://www.ris.bka.gv.at/eli/bgbl/II/2016/219/20160809 (28.04.2021).

BMBF - Bundesministerium für Bildung und Frauen (Hg.) (2014): Unterrichtsprinzip Medienerziehung - Grundsatzerlass. https://www.bmbwf.gv.at/Themen/schule/ schulrecht/rs/1997-2017/2012_04.html (28.04.2021).

BMBWF - Bundesministerium für Bildung, Wissenschaft und Forschung (Hg.) (2018): Verordnung des Bundesministers für Bildung, Wissenschaft und Forschung, mit der die Verordnung über die Lehrpläne der Neuen Mittelschulen sowie die Verordnung über die Lehrpläne der allgemeinbildenden höheren Schulen geändert werden. Wien. BGBl. II Nr. 71/2018 vom 19.04.2018. https:// www.ris.bka.gv.at/Dokumente/BgblAuth/BGBLA_2018_ II_71/BGBLA_2018_II_71.pdfsig (28.04.2021).

Bogner, K. \& U. Landrock (2015): Antworttendenzen in standardisierten Umfragen. GESIS - Leibniz-Institut für Sozialwissenschaften, Mannheim.

Callegaro, M., K. Lozar Manfreda \& V. Vehovar (2015): Web Survey Methodology. SAGE, London. 
Carling, J. \& F. Collins (2018): Aspiration, Desire and Drivers of Migration. In: Journal of Ethnic and Migration Studies 44(6). S. 909-926.

Collins, F., R. Sidhu, N. Lewis \& B. Yeoh (2014): Mobility and Desire: International Students and Asian Regionalism in Aspirational Singapore. In: Discourse: Studies in the Cultural Politics of Education 35(5). S. 661-676.

Creswell, J. \& V. Plano Clark (2007): Designing and Conducting Mixed Methods Research. SAGE, Thousand Oaks.

DGfG - Deutsche Gesellschaft für Geographie (Hg.) (2020): Bildungsstandards im Fach Geographie für den Mittleren Schulabschluss mit Aufgabenbeispielen. 10. Aufl. DGfG, Bonn.

Evans, J. R. \& A. Mathur (2018): The value of online surveys: a look back and a look ahead. In: Internet Research 28(4). S. 854-887.

Glantz, A. \& T. Michael (2014): Interviewereffekte. In: Baur, N. \& J. Blasius (Hrsg.): Handbuch Methoden der empirischen Sozialforschung. Springer VS, Wiesbaden. S. 313-322.

Hammer, V. \& M. Knopp (2015): Datenschutzinstrumente. Anonymisierung, Pseudonyme und Verschlüsselung. In: DuD. Datenschutz und Datensicherheit 39. S. 503-509.

Hansen, H. K. \& L. G. Aner (2017): On the location dynamics of highly educated people migrating to peripheral regions of Denmark. In: Population, Space and Place 28(8). S. 1-12.

Hinsch, S., H. Pichler, T. Jekel, L. Keller \& F. Baier (2014): Semestrierter Lehrplan AHS, Sekundarstufe II. Ergebnis der ministeriellen Arbeitsgruppe. In: GW-Unterricht 136(4). S. 51-61.

Hollenberg, S. (2016): Fragebögen. Fundierte Konstruktion, sachgerechte Anwendung und aussagekräftige Auswertung. Springer VS, Wiesbaden.

Jandura, O. \& M. Leidecker (2013): Grundgesamtheit und Stichprobenbildung. In: Möhring, W. \& D. Schlütz (Hrsg.): Handbuch standardisierte Erhebungsverfahren in der Kommunikationswissenschaft. Springer VS, Wiesbaden. S. 61-78.

Jonkisz, E., H. Moosbrugger, \& H. Brandt (2012): Planung und Entwicklung von Tests und Fragebogen. In: Moosbrugger, H. \& A. Kelava (Hrsg.): Testtheorie und Fragebogenkonstruktion. Springer, Heidelberg. S. 27-74.

Kanwischer, D. (2011): Kompetenzorientierung im Geographieunterricht. Von den Leitgedanken zur Praxis. In: GW-Unterricht 122(2). S. 3-16.

Kirchhoff, B., S. Kuhnt, P. Lipp \& S. Schlawin (2010): Der Fragebogen. Datenbasis, Konstruktion und Auswertung. Springer VS, Wiesbaden.

Kühne, R. (2013): Konzeptspezifikation und Messung. In: Möhring, W. \& D. Schlütz (Hrsg.): Handbuch standardisierte Erhebungsverfahren in der Kommunikationswissenschaft. Springer VS, Wiesbaden. S. 23-40.

Leibert, T. (2016): She leaves, he stays? Sex-selective migration in rural East Germany. In: Journal of Rural Studies 43. S. 267-279.
Meier Kruker, V. \& J. Reuh (2005): Arbeitsmethoden der Humangeographie. WBG, Darmstadt.

Meyer, F. (2017): Navigating aspirations and expectations: adolescents' considerations of outmigration from rural eastern Germany. In: Journal of Ethnic and Migration Studies 44(6). S. 1032-1049.

Meyer, F., K. Beurskens \& J. Miggelbrink (2019): Looping Effects and Empirical Fieldwork: Tackling the Complexity of Adolescents' Migratory Aspirations in Rural Eastern Germany through Focus Groups and Interviews. In: SAGE Research Methods Cases Part 2.

Meyer, F. \& T. Leibert (2021): "You have to leave some day!" On the role of cultures of migration in the migration decisions of young people in shrinking regions of Central Germany. In: Geographica Helvetica 76. S. 335-345.

Meyer, F., J. Miggelbrink, J. \& T. Schwarzenberg (2016): Reflecting on the Margins: Socio-spatial Stigmatisation among Adolescents in a Peripheralised Region. In: Comparative Population Studies 41(3-4). S. 285-320.

Nayak, M. S. D. P. \& K. A. Narayan (2019): Strengths and Weakness of Online Surveys. In: IOSR Journal of Humanities and Social Science 24(5). S. 31-38.

Padberg, S. (2012): Geographie kritisch und themenzentriert unterrichten und was das mit der Debatte um Kompetenzorientierung zu tun hat. In: GW-Unterricht 127(3). S. 12-28.

Pichler, H. (2013): Kritische Kompetenzorientierung konkret. Fachdidaktische Leitgedanken für die Umsetzung einer kritisch gewendeten Kompetenzorientierung im GW-Unterricht und für die Erstellung von Aufgaben für die kompetenzorientierte Reife- und (Diplom-)Prüfung. In: GW-Unterricht 130(2). S. 15-22.

Pichler, H., C. Fridrich, C. Vielhaber \& F. Bergmeister (2017): Der fachdidaktische Grundkonsens 2.0 in der Verbundregion Nordost. Perspektiven einer zukunftsfähigen Orientierungshilfe im GW-Unterricht. In: GWUnterricht 146(2). S. 60-62.

Porst, R. (2014): Fragebogen. Ein Arbeitsbuch. Springer VS, Wiesbaden.

RatSWD (2017): Handreichung Datenschutz. Rat für Sozial- und Wirtschaftsdaten (RatSWD), Berlin.

Reinecke, J. (2014): Grundlagen der standardisierten Befragung. In: Baur, N. \& J. Blasius (Hrsg.): Handbuch Methoden der empirischen Sozialforschung. Springer VS, Wiesbaden. S. 601-618.

Reusser, K. (2005): Problemorientiertes Lernen. Tiefenstruktur, Gestaltungsformen, Wirkung. In: Beiträge zur Lehrerinnen- und Lehrerbildung 23(2). S. 159-182.

Steiner, E. \& M. Benesch (2018): Der Fragebogen. Von der Forschungsidee zur SPSS-Auswertung. UTB, Wien.

Taddicken, M. (2013): Online-Befragung. In: Möhring, W. \& D. Schlütz (Hrsg.): Handbuch standardisierte Erhebungsverfahren in der Kommunikationswissenschaft. Springer VS, Wiesbaden. S. 201-217.

Tillmann A. (2015): Forschendes Lernen im Geographieunterricht. In: Gebhard U. (Hg.): Sinn im Dialog. Springer VS, Wiesbaden. S. 235-252. 
Vielhaber, C. (1999): Vermittlung und Interesse - Zwei Schlüsselkategorien fachdidaktischer Grundlegungen im Geographieunterricht. In: Christian Vielhaber (Hrsg.): Geographiedidaktik kreuz und quer. Vom Vermittlungsinteresse bis zum Methodenstreit - Von der Spurensuche bis zum Raumverzicht. Selbstverlag des Instituts für Geographie der Universität Wien. Wien, S. 9-26. (= Materialien zur Didaktik der Geographie und Wirtschaftskunde 15)

Vogler, R., T. Jekel \& E. Killingseder (2018): Using the geographies of learning. An exploratory categorization for spatially enabled learning. In: GI_Forum 2018 Journal for Geographic Information Science 2018(2). S. 181-192.

Wagner, P. \& L. Hering (2014): Online-Befragung. In: Baur, N. \& J. Blasius (Hrsg.): Handbuch Methoden der empirischen Sozialforschung. Springer VS, Wiesbaden. S. 661-674.

Wang, A. I. \& R. Tahir (2020): The effect of using Kahoot! for learning - A literature review. In: Computers \& Education 149(7). Art. 103818.

Weichbold, M. (2014): Pre-Test. In: Baur, N. \& J. Blasius (Hrsg.): Handbuch Methoden der empirischen Sozialforschung. Springer VS, Wiesbaden. S. 299-304.

Welker, M. \& C. Wünsch (2010): Methoden der OnlineForschung. In: Schweiger, W. \& K. Beck (Hrsg.): Handbuch Online-Kommunikation. Springer VS, Wiesbaden. S. 487-517.

Wiest, K. (2016): Migration and everyday discourses: Peripheralisation in rural Saxony-Anhalt from a gender perspective. In: Journal of Rural Studies 43. S. 280-290. 


\section{Anhang}

\section{A: Exemplarischer Fragebogen zu Wegzugmotiven Jugendlicher ${ }^{7}$}

Frage 1: Haben Sie sich schon einmal mit der Frage befasst, nach Ihrem Schulabschluss aus Ihrer Heimatregion / -stadt wegzuziehen?
$\mathrm{O}$ ja
nein

Frage 2: Welche Kriterien sind in Ihren Augen bei dieser Entscheidung besonders relevant?

(Mehrfachantworten möglich)

O Ausbildungsmöglichkeiten (bspw. Studium, Berufsausbildung, Schule)

O Erreichbarkeit (bspw. ÖPNV-Angebot)

O Freizeitmöglichkeiten (bspw. Konsum, Kino, Sport)

O private Gründe (bspw. Partnerschaft, familiäre Gründe)

Negative Erfahrungen am Heimatort

Positive Erfahrungen am Zielort

Andere Gründe:

Frage 3: In welchen Kreisen haben Sie dieses Thema bisher am häufigsten diskutiert? (Einfachantwort)
O Schule/ Klassenverband
Freundeskreis
Familie

O Andere, und zwar

O Ich habe darüber bisher noch nicht mit anderen Personen gesprochen.

Frage 4: Wenn in Ihrer Familie über das Thema des Wegzuges gesprochen wird, mit wem sprechen Sie darüber? (Mehrfachantworten möglich)
Oltern
O Großeltern
Geschwister
O Andere:

Frage 5: Bitte geben Sie bei den folgenden Aussagen zur Rolle der Familie an, inwieweit diese zutreffen. (ein Kreuz pro Zeile)

\begin{tabular}{|c|c|c|c|c|c|}
\hline Aussage & Trifft zu & $\begin{array}{c}\text { Trifft eher } \\
\text { zu }\end{array}$ & $\begin{array}{l}\text { Trifft eher } \\
\text { nicht zu }\end{array}$ & $\begin{array}{c}\text { Trifft } \\
\text { nicht zu }\end{array}$ & $\begin{array}{l}\text { Ich weiß } \\
\text { es nicht }\end{array}$ \\
\hline \multicolumn{6}{|l|}{$\begin{array}{l}\text { In meiner Familie wird über meine Pläne nach dem } \\
\text { Schulabschluss diskutiert. }\end{array}$} \\
\hline \multicolumn{6}{|l|}{ In meiner Familie wird über Wegzug diskutiert. } \\
\hline \multicolumn{6}{|l|}{ Meine Familie unterstützt es, wenn ich wegziehe. } \\
\hline \multicolumn{6}{|l|}{$\begin{array}{l}\text { In meiner Familie gibt es unterschiedliche Meinungen zu } \\
\text { Wegzügen. }\end{array}$} \\
\hline \multicolumn{6}{|l|}{ Meiner Familie ist meine berufliche Zukunft wichtig. } \\
\hline \multicolumn{6}{|l|}{$\begin{array}{l}\text { Meiner Familie ist mein Verbleib in meinem Landkreis/ } \\
\text { Bezirk wichtig. }\end{array}$} \\
\hline \multicolumn{6}{|l|}{ Meine Eltern haben Erfahrungen mit Wegzügen. } \\
\hline $\begin{array}{l}\text { Meine Geschwister oder andere gleichaltrige Verwandte } \\
\text { haben Erfahrungen mit Wegzügen. }\end{array}$ & & & & & \\
\hline
\end{tabular}

\footnotetext{
Wir bedanken uns herzlich bei Tom Schwarzenberg (TU Dresden) und Tim Leibert (Ifl Leipzig) für die konstruktiven Kommentare zum Fragebogenentwurf.
} 
Frage 6: Bitte geben Sie bei den folgenden Aussagen zur Rolle der Schule/ des Klassenverbandes an, inwieweit diese zutreffen. (ein Kreuz pro Zeile)

\begin{tabular}{|c|c|c|c|c|c|}
\hline Aussage & Trifft zu & $\begin{array}{c}\text { Trifft eher } \\
\text { zu }\end{array}$ & $\begin{array}{l}\text { Trifft eher } \\
\text { nicht zu }\end{array}$ & $\begin{array}{c}\text { Trifft } \\
\text { nicht zu }\end{array}$ & $\begin{array}{l}\text { Ich weiß } \\
\text { es nicht }\end{array}$ \\
\hline $\begin{array}{l}\text { In meiner Schule wird innerhalb des Unterrichts über } \\
\text { Pläne nach dem Schulabschluss diskutiert. }\end{array}$ & & & & & \\
\hline $\begin{array}{l}\text { In meiner Schule wird innerhalb des Unterrichts über } \\
\text { Wegzug diskutiert. }\end{array}$ & & & & & \\
\hline $\begin{array}{l}\text { In meiner Schule wird in den Pausen über Pläne nach } \\
\text { dem Schulabschluss diskutiert. }\end{array}$ & & & & & \\
\hline $\begin{array}{l}\text { In meiner Schule wird in den Pausen über Wegzug disku- } \\
\text { tiert. }\end{array}$ & & & & & \\
\hline $\begin{array}{l}\text { Während meiner Schulzeit habe ich Praktika in meiner } \\
\text { Heimatregion abgeleistet. }\end{array}$ & & & & & \\
\hline $\begin{array}{l}\text { Während meiner Schulzeit habe ich Praktika außerhalb } \\
\text { meiner Heimatregion abgeleistet. }\end{array}$ & & & & & \\
\hline $\begin{array}{l}\text { Über die Schule wurden Berufsinformationsveranstaltun- } \\
\text { gen organisiert. }\end{array}$ & & & & & \\
\hline
\end{tabular}

Frage 7: Bitte geben Sie bei den folgenden Aussagen zur Rolle des Freundeskreises an, inwieweit diese zutreffen. (ein Kreuz pro Zeile)

\begin{tabular}{|c|c|c|c|c|c|}
\hline Aussage & Trifft zu & $\begin{array}{c}\text { Trifft eher } \\
\text { zu }\end{array}$ & $\begin{array}{l}\text { Trifft eher } \\
\text { nicht zu }\end{array}$ & $\begin{array}{c}\text { Trifft } \\
\text { nicht zu }\end{array}$ & $\begin{array}{l}\text { Ich weiß } \\
\text { es nicht }\end{array}$ \\
\hline $\begin{array}{l}\text { In meinem Freundeskreis wird über Pläne nach dem } \\
\text { Schulabschluss diskutiert. }\end{array}$ & & & & & \\
\hline In meinem Freundeskreis wird über Wegzug diskutiert. & & & & & \\
\hline $\begin{array}{l}\text { In meinem Freundeskreis gibt es Erfahrungen mit Weg- } \\
\text { zügen. }\end{array}$ & & & & & \\
\hline $\begin{array}{l}\text { In meinem Freundeskreis ist es normal, nach dem Schul- } \\
\text { abschluss wegzuziehen. }\end{array}$ & & & & & \\
\hline $\begin{array}{l}\text { In meinem Freundeskreis wird es als normal angesehen, } \\
\text { nach dem Schulabschluss in der Heimatregion zu bleiben. }\end{array}$ & & & & & \\
\hline $\begin{array}{l}\text { In meinem Freundeskreis tauschen wir uns über Berufser- } \\
\text { fahrungen (bspw. Praktika) aus. }\end{array}$ & & & & & \\
\hline
\end{tabular}

Frage 8: Mit welchen Begriffen verbinden Sie den Gedanken an Wegzug? Machen Sie hierzu ein Kreuz zwischen den beiden Polen.

$\begin{array}{rllllll}\text { selbstbestimmt } & \bigcirc & 0 & 0 & 0 & 0 & \text { gezwungen } \\ \text { beengend } & \bigcirc & 0 & 0 & 0 & \bigcirc & \text { erfüllend } \\ \text { emotional } & 0 & 0 & 0 & 0 & 0 & \text { pragmatisch } \\ \text { traurig } & 0 & 0 & 0 & 0 & 0 & \text { glücklich } \\ \text { einfach } & 0 & 0 & 0 & 0 & 0 & \text { schwer } \\ \text { bedeutend } & 0 & 0 & 0 & 0 & 0 & \text { unbedeutend }\end{array}$

Frage 9: Hat das Image Ihrer Heimatregion eine Auswirkung auf Ihre Wanderungsentscheidung? (Offene Frage)

Frage 10: Welche Schulform besuchen Sie aktuell?
DE: $\quad O$ Gymnasium
O Realschule
O Berufsschule
andere
AT: $\quad$ O AHS
$\mathrm{O}$ BHS
O MS
$O$ andere

Frage 11: In welcher Klassenstufe befinden Sie sich?
Klasse 9 oder 10
Klasse 11,12 oder 13
$\mathrm{O}$ andere

Frage 12: Welches Geschlecht haben Sie?
männlich
O weiblich
O divers 


\section{B: Exemplarische Erstzusammenfassung mittels Google Forms}

Die folgenden Illustrationen entsprechen einer direkten und unmittelbaren Ergebnispräsentation des in Google Forms umgesetzten Fragebogens aus Anhang A in der Benutzeroberfläche der Plattform ohne weitere Bearbeitungen. Die erhobenen Daten können aber auch als Datensatz (Excel Tabelle) exportiert und differenzierter analysiert werden. Dementsprechend sind alternative und teils geeignetere Visualisierungsformen mit externen Programmen umsetzbar.

Hinweis: Da es sich bei dieser Illustration um eine (der im Artikel beschriebenen Unterrichtssequenz folgend) beispielhafte klasseninterne Erhebung handelt, wurden die Fragen 10 und 11 aus dem Beispielfragebogen aus Anhang A ausgelassen.

Haben Sie sich schon einmal mit der Frage befasst, nach Ihrem Schulabschluss aus Ihrer Heimatregion / -stadt wegzuziehen?

25 Antworten
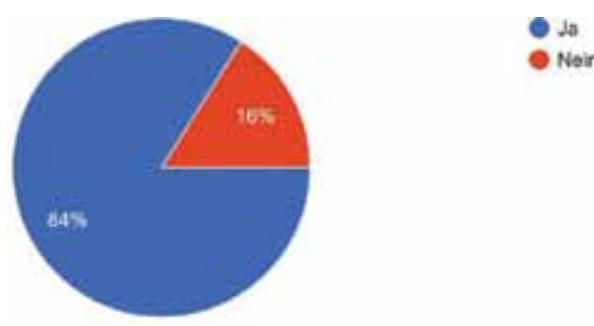

Welche Kriterien sind in Ihren Augen bei dieser Entscheidung besonders relevant? (Mehrfachantworten möglich)

25 Antworten

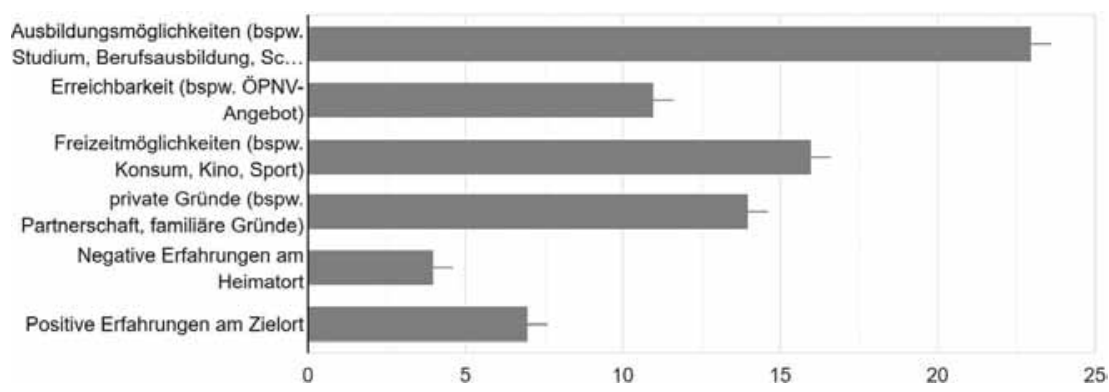

In welchen Kreisen haben Sie dieses Thema bisher am häufigsten diskutiert?

25 Antworten

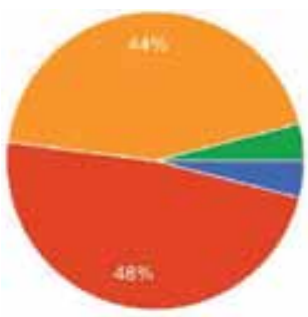

- Schule/Klassamertand

- Freundeskreis

- Fandie

- ich habe daruber bisher noch richt mit anderen Porsonen gusprochen

Wenn in Ihrer Familie über das Thema des Wegzuges gesprochen wird, mit wem sprechen Sie darüber (Mehrfachantworten möglich)

24 Antworten

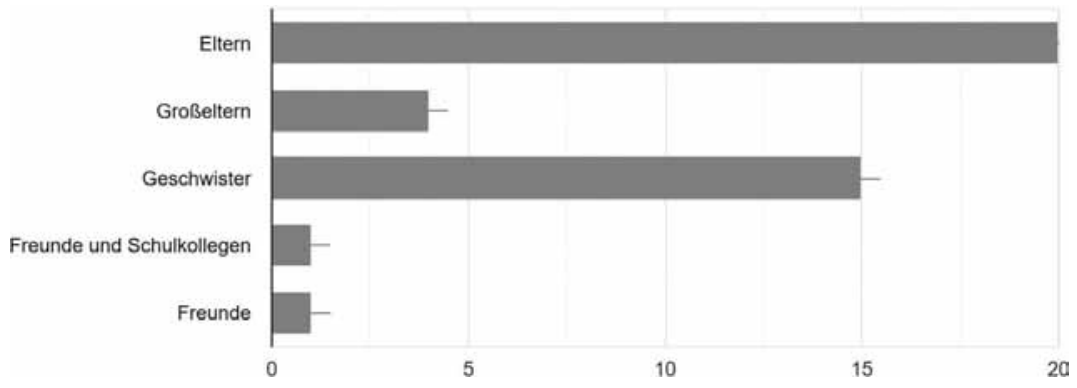


Bitte geben Sie bei den folgenden Aussagen zur Rolle der Familie an, inwieweit diese zutreffen.

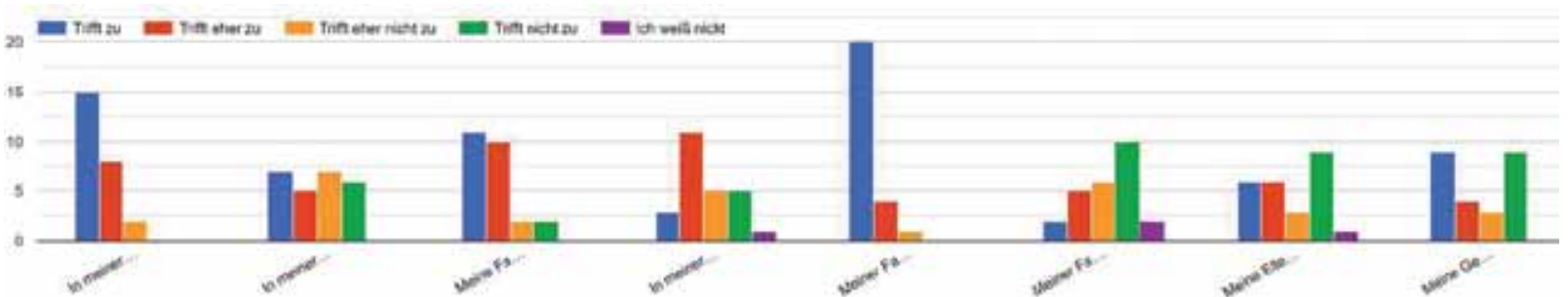

Bitte geben Sie bei den folgenden Aussagen zur Rolle der Schule/des Klassenverbandes an, inwieweit diese zutreffen.

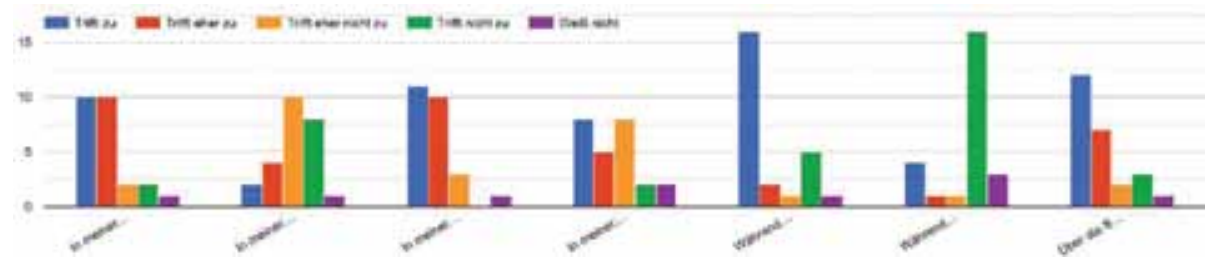

Bitte geben Sie bei den folgenden Aussagen zur Rolle des Freundeskreises an, inwieweit diese zutreffen.

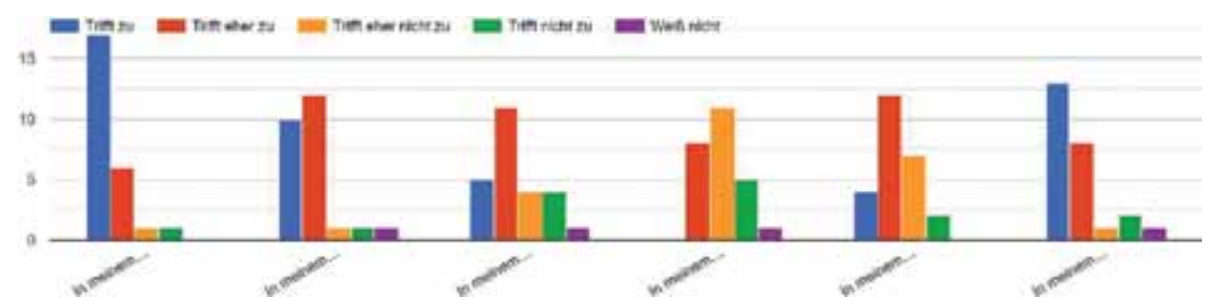

Mit welchen Begriffen verbinden Sie den Gedanken an Wegzug? Machen Sie hierzu ein Kreuz zwischen den beiden Polen.

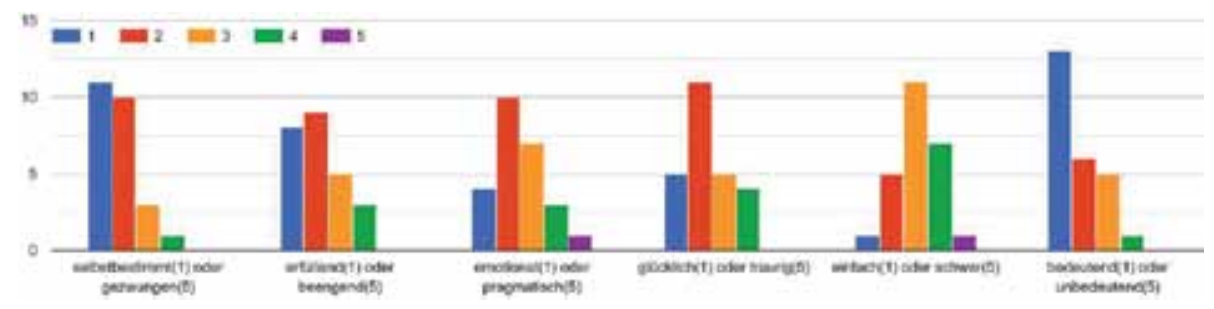

Welches Geschlecht haben Sie?

25 Antworten

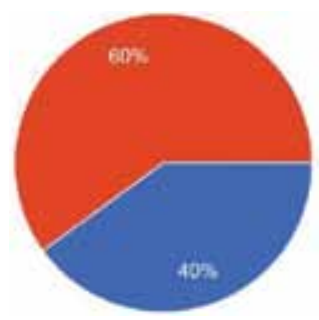

